\title{
Perfil dos idosos residentes em uma instituição de longa permanência
}

\author{
Profile of elderly living in a long-term care institution
}

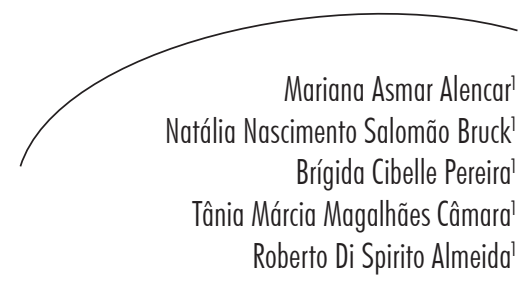

\section{Resumo}

Objetivo: Traçar o perfil clínico-funcional de idosos de uma instituição de longa permanência para idosos (ILPI). Métodos: Participaram 47 idosos de uma ILPI de Belo Horizonte/MG. Utilizaram-se questionário com informações sociodemográficas, Mini Exame do Estado Mental (MEEM), Escala de Depressão Geriátrica (GDS), Índice de Katz e Time Up and Go (TUG). Realizou-se análise descritiva (programa SPSS 14.0). Resultados: Os idosos apresentaram média de 73,0 anos $( \pm 8,99)$. A maioria era mulher $(51,1 \%)$, solteira $(46,8 \%)$, sem filhos $(51,1 \%)$ e de baixa escolaridade. O tempo de institucionalização variou de um mês a 25 anos. O motivo de institucionalização mais frequente foi para tratamento $(29,8 \%$ ), e $80,9 \%$ relataram receber visitas. Os idosos tomam em média 3,45 medicamentos $( \pm 1,87)$. Apenas $12,8 \%$ relataram quedas nos últimos seis meses e $80,9 \%$ tinham medo de cair. Cerca de 38,3\% não utilizam dispositivo de auxílio à marcha, 12,8\% utilizavam cadeira de rodas, 42,5\% andador e 6,4\% bengala. O desempenho no MEEM foi baixo, sendo que 93,3\% apresentaram valores abaixo da nota de corte para escolaridade. Na GDS, 59,6\% obtiveram valores que sugerem quadro depressivo. O TUG variou de 0,10 a 1,58 minutos. A maioria relatou ser independente para realizar as atividades do Katz (banhar-se: 80,9\%; vestirse: $83,0 \%$; usar banheiro: $80,9 \%$; transferir-se: $87,2 \%$; micção: 59,6\%; evacuação: $61,7 \%$; alimentar-se: 93,6\%) e nenhum relatou dependência completa. Conclusões: O perfil da população de idosos institucionalizados está de acordo com a literatura nos aspectos clínico-demográficos. A maioria dos idosos, entretanto, relatou ser independente para as atividades básicas de vida diária, o que representa boa oportunidade para intervenções que visem prevenir ou minimizar possíveis perdas funcionais.

\section{Abstract}

Objective: To describe the clinical and functional profile of elderly living in a long-term care institution. Methods: 47 older adults living in a long-term care institution of Belo Horizonte/MG took part in the study. For a data collection were used a structured socio-demographic questionnaire, Mini Mental State Examination (MMSE), Geriatric

Palavras-chave: Idoso. Instituição de Longa Permanência para Idoso. Perfil clínico-funcional.

Faculdade de Ciências Sociais, Humanas e da Saúde. Universidade FUMEC. Belo Horizonte, MG, Brasil. 
Depression Scale(GDS), Katz Index and Timed Up and Go (TUG). A descriptive statistics were employed by using SPSS14.0. Results: The mean age of the elderly was 73.0 years $( \pm 8.99)$. Most of the elderly was female $(51.1 \%)$, single $(46.8 \%)$ without children (51.1\%) and with low education. The time of institutionalization ranged from one month to 25 years. The most frequent reason for institutionalization was with purpose of treatment $(29.8 \%)$ and majority reported receiving visits $(80.9 \%)$. The elderly took an average of 3.45 medications $( \pm 1.87)$. Only $12.8 \%$ reported at least one fall in the last six months and $80.9 \%$ reported being afraid of falling. About $38.3 \%$ do not use assistive walk device; $12.8 \%$ use wheelchair; $42.5 \%$, walker; and $6.4 \%$, cane. The MMSE scores were low, 93.3\% were below the cutoff score for their schooling. At GDS, 59.6\% were suggesting possible depression. TUG range from 0.10 to 1.58 minutes. Most elderly people reported being independent to perform the activities of Katz (bathing: 80.9\%; dressing: $83.0 \%$; toileting: $80.9 \%$; transferring $87.2 \%$; urination $59.6 \%$; defecation: $61.7 \%$; feeding $93.6 \%$ ) and none reported complete dependence. Conclusion: The profile of institutionalized elderly is consistent with the literature on clinical and demographic variables. However, most patients reported being independent for basic activities of daily living, which represents a good opportunity for interventions that aim to prevent or minimize possible losses of function.

\section{INTRODUÇÃO}

Nas últimas décadas, o cenário demográfico mundial vem sendo caracterizado pelo envelhecimento populacional. ${ }^{1}$ Como contribuição a este fenômeno, o Brasil tem apresentado um dos mais rápidos crescimentos da população idosa. Além do aumento no número de idosos, tem havido acréscimo significativo na expectativa de vida da população brasileira. Esta, que era em torno de 33,7 anos em 1950/1955, passou para 50,99 em 1990, chegou até 66,25 em 1995, 71,7 anos em 2004 e alcançou os 73,5 anos em 2010. ${ }^{1}$

Para muitos idosos, infelizmente, esse aumento da longevidade tem sido acompanhado de um declínio do estado de saúde físico e mental, presença de múltiplas doenças crônicas, perda de independência e autonomia, e limitações socioeconômicas e ambientais, que são fatores associados à limitação da capacidade funcional dos idosos. ${ }^{2}$

A redução dessa capacidade funcional, ou seja, a presença de dificuldade ou dependência na realização de atividades essenciais para uma vida independente, incluindo atividades de autocuidado e aquelas consideradas importantes para a qualidade de vida do indivíduo, ${ }^{3,4}$ é um achado frequente entre os idosos no Brasil., ${ }^{2,3}$
Key words: Aged. Homes for the Aged. Clinical and functional profile.
Resultados de pesquisa no município de São Paulo mostraram que mais da metade da população estudada $(53 \%)$ referia necessidade de ajuda parcial ou total para realizar pelo menos uma atividade da vida diária (AVD). Foi detectado, também, que $29 \%$ dos idosos necessitam de ajuda parcial ou total para realizar até três dessas atividades, e 17\% necessitavam de ajuda para realizar quatro ou mais AVDs., ${ }^{2,3}$

Esta perda funcional se torna um fator de risco para a institucionalização, que se acentuou com a mudança da dinâmica familiar e da sociedade nos últimos anos. ${ }^{5,6}$ Paralelamente a isso, ainda existe a incapacidade da família de encontrar alguém que se responsabilize pelo cuidado do idoso. Aumenta, então, a procura de instituições de longa permanência para idosos (ILPI) que ofereçam cuidados necessários para o idoso, suprindo a falta de suporte familiar e social. ${ }^{6,7}$

De acordo com Davim et al. ${ }^{8}$ e Perlini et al., ${ }^{6}$ mesmo um idoso institucionalizado deve ter o cuidado de preservar e/ou melhorar sua qualidade de vida, autonomia e independência. No entanto, muitas vezes o que ocorre em uma instituição, com o intuito de agilizar o processo de atendimento, é a diminuição do incentivo a esses idosos para continuarem realizando independentemente suas atividades, o que 
pode levar a uma piora do quadro funcional e limitação, tornando mais difícil o trabalho da equipe multidisciplinar na avaliação e no tratamento do idoso.

Apesar do grande número de ILPIs no Brasil, existem poucos estudos verificando o perfil funcional desses idosos. Este fato dificulta a implementação de programas de assistência a esta população e, ainda, de orientação e treinamento dos profissionais envolvidos no seu cuidado. Portanto, o objetivo do presente estudo foi caracterizar o perfil clínico-funcional dos idosos que vivem em uma instituição de longa permanência localizada em Belo Horizonte, Minas Gerais.

\section{MATERIAIS E MÉTODOS}

\section{Questões éticas}

O presente estudo foi aprovado pelo Comitê de Ética em Pesquisa da Universidade FUMEC (CEP no 207/2008). Os idosos e seus responsáveis receberam explicação detalhada sobre os objetivos e procedimentos de avaliação e assinaram o termo de consentimento livre e esclarecido, concordando em participar voluntariamente da pesquisa.

\section{Amostra}

A amostra foi de conveniência e constituída por 47 idosos - 24 mulheres e 23 homens -, com idade igual ou superior a 60 anos, média de 73,0 anos $( \pm 8,99)$ e residentes em uma instituição de longa permanência de Belo Horizonte, Minas Gerais. Foram excluídos os idosos que apresentassem alterações visuais e/ou auditivas graves, acamados, com instabilidade clínica ou demência grave, segundo os critérios da Escala Clínica de Demência (Clinical Dementia Rating - CDR).

\section{Instrumentos}

As informações clínicas, sociodemográficas e descritivas do perfil do idoso foram coletas utilizando-se um formulário semiestruturado e por autorrelato do idoso ou cuidador. ${ }^{10}$

Para avaliar os aspectos funcionais relacionados às AVDs, foi utilizado o Índice de Katz. Essa escala já foi validada e adaptada para a população brasileira e tem por objetivo avaliar o grau de dependência dos idosos relacionados às atividades básicas de vida diária (ABVDs), como: tomar banho, vestirse, ir ao banheiro, realizar transferências, ter continência e alimentar-se. ${ }^{11} \mathrm{O}$ Índice de Katz foi avaliado pelo autorrelato do paciente..$^{11,12}$

Para se avaliar a mobilidade funcional por meio do desempenho, utilizou-se o Timed Up and Go (TUG). O objetivo do TUG é avaliar o equilíbrio sentado, transferência de sentado para de pé, estabilidade na marcha e mudança do percurso na deambulação. ${ }^{10} \mathrm{O}$ teste mede em segundos o tempo gasto, pelo indivíduo, levantar de uma cadeira, andar uma distância de três metros, virar $180^{\circ}$, retornar e sentar novamente na cadeira. ${ }^{13}$ Para a realização do teste, foi utilizada uma cadeira com braços (altura do assento de $45 \mathrm{~cm}$ e dos braços de $65 \mathrm{~cm})^{10,13}$

Foi utilizada a Escala de Depressão Geriátrica (Geriatric Depression Scale - GDS), versão curta (GDS-15), para avaliar a presença ou não de sintomas depressivos. ${ }^{14,15}$ A GDS é um instrumento criado por Yesavage et al. ${ }^{14} \mathrm{e}$ teve sua adaptação e tradução para português no Brasil. ${ }^{14}$ É frequentemente utilizado na Geriatria para rastrear transtornos depressivos. A versão brasileira da GDS 15, com ponto de corte $5 / 6$, produziu índices de sensibilidade de $85,4 \%$ e especificidade de $73,9 \%$, para rastreio de episódio depressivo maior, de acordo com a Classificação Internacional de Doenças (CID -10) e confiabilidade teste e reteste moderada. ${ }^{15}$

A função cognitiva foi avaliada pelo Mini Exame do Estado Mental (MEEM), que avalia a presença ou não de possíveis alterações cognitivas. É um instrumento bastante utilizado e envolve duas categorias de resposta -verbais e não verbais. É composto por seis itens que avaliam: orientação temporal e espacial, registro, memória imediata, cálculo, memória recente e linguagem. Possui um escore máximo de 30 pontos. Segundo Lourenço et al., ${ }^{16}$ os fatores que irão determinar a presença 
de déficits cognitivos ou não serão os seguintes valores de corte: 18 para analfabetos (sensibilidade $=73,5 \%$; especificidade $=73,9 \%)$ e 24 para pessoas com instrução escolar (sensibilidade $=$ $75 \%$; especificidade $=69,7 \%) .{ }^{16}$

\section{Procedimentos de análise}

Foi realizada análise descritiva dos dados utilizando o programa estatístico Statistical Package for the Social Sciences (SPSS) versão 14.0. Foram utilizadas estatísticas descritivas básicas, como: frequência, média, mediana, desvio padrão e valor mínimo e máximo.

\section{RESULTADOS}

Foram avaliados 47 idosos, sendo 24 mulheres $(51,1 \%)$ e 23 homens (48,9\%), com idade variando de 60 a 92 anos (73,0 $\pm 8,9$ anos). A maioria era solteira ( $\mathrm{n}=22 ; 46,8 \%)$ e de baixa escolaridade, sendo $25,5 \%$ analfabetos e $55,3 \% \leq 4$ anos de estudo. Um pouco mais da metade relatou não possuir filhos $(\mathrm{n}=24$; $51,1 \%$ ), de acordo com a tabela 1.

Tabela 1 - Descrição do perfil sociodemográfico. Belo Horizonte-MG, 2009.

\begin{tabular}{|c|c|}
\hline Variáveis & (n) $\%$ \\
\hline \multicolumn{2}{|l|}{ Sexo } \\
\hline Feminino & (24) $51,1 \%$ \\
\hline Masculino & (23) $48,9 \%$ \\
\hline \multicolumn{2}{|l|}{ Estado civil } \\
\hline Solteiros & (22) $46,8 \%$ \\
\hline Viúvos & (16) $34,0 \%$ \\
\hline Casados & (8) $17,1 \%$ \\
\hline Separados/Divorciados & (1) $2,1 \%$ \\
\hline \multicolumn{2}{|l|}{ Escolaridade } \\
\hline Analfabeto & (12) $25,5 \%$ \\
\hline$\leq 4$ anos de estudo & (26) $55,3 \%$ \\
\hline$>4$ anos de estudo & (9) $19,2 \%$ \\
\hline \multicolumn{2}{|l|}{ Ter filhos } \\
\hline Não & (24) $51,1 \%$ \\
\hline Sim & (23) $48,9 \%$ \\
\hline \multicolumn{2}{|l|}{ Motivo da institucionalização } \\
\hline Tranquilidade & (3) $6,4 \%$ \\
\hline Tratamento & (14) $29,8 \%$ \\
\hline Idade & (1) $2,1 \%$ \\
\hline Morava sozinho & (3) $6,4 \%$ \\
\hline Motivos financeiros & (1) $2,1 \%$ \\
\hline Não tinha onde morar & (4) $8,5 \%$ \\
\hline Doença & (7) $14,9 \%$ \\
\hline Filho, neto ou cônjuge trouxe & (4) $8,5 \%$ \\
\hline Achou melhor & (1) $2,1 \%$ \\
\hline Ficou sem família & (1) $2,1 \%$ \\
\hline Não soube responder & (8) $17,1 \%$ \\
\hline \multicolumn{2}{|l|}{ Recebimento de visitas } \\
\hline Não & (8) $17,1 \%$ \\
\hline $\operatorname{Sim}$ & (38) $80,9 \%$ \\
\hline Não soube responder & (1) $2 \%$ \\
\hline
\end{tabular}

$\mathrm{n}=$ número de idosos; $\%=$ percentagem de idosos 
O motivo de institucionalização mais frequente foi para tratamento $(29,8 \%)$ e a maioria relatou receber visitas $(80,9 \%)$, segundo a tabela 1. O tempo de institucionalização dos idosos variou de um mês a 25 anos (mediana: 4,0 anos).

A maioria dos idosos $(57,4 \% ; \mathrm{n}=27)$ referiu ter pelo menos uma doença crônica e 91,5\%
( $n=43)$ fazem uso de pelo menos uma medicação. Os idosos utilizavam, em média, 3,45 $( \pm 1,87)$ medicamentos, sendo a variação de um a sete medicamentos. A autopercepção de saúde de $61,7 \%$ ( $\mathrm{n}=29$ ) dos idosos foi boa. Um pouco mais da metade dos idosos, $51,1 \%(\mathrm{n}=24)$, relatou sentir algum tipo de dor crônica (tabela 2).

Tabela 2 - Descrição das características clínico-funcionais. Belo Horizonte-MG, 2009.

\begin{tabular}{lc}
\hline \multicolumn{1}{c}{ Variáveis } & $(\mathrm{n}) \%$ \\
\hline Possuir doenças crônicas & \\
Não & $(15) 31,9 \%$ \\
Sim & $(27) 57,4 \%$ \\
Não souberam responder & $(5) 10,7 \%$ \\
Uso de medicamentos & \\
Não & $(4) 8,5 \%$ \\
Sim & $(43)) 91,5 \%$ \\
Auto-percepção de saúde & \\
Ruim & $(3) 6,4 \%$ \\
Regular & $(10) 21,3 \%$ \\
Boa & $(29) 61,7 \%$ \\
Muito boa & $(1) 2,1 \%$ \\
Ótima & $(4) 8,5 \%$ \\
Relato de dor & \\
Não & $(23) 48,9 \%$ \\
Sim & $(24) 51,1 \%$ \\
Quedas nos últimos 6 meses & \\
Não & $(41) 87,2 \%$ \\
Sim & $(6) 12,8 \%$ \\
Medo de quedas & \\
Não & $(9) 19,1 \%$ \\
Um pouco & $(8) 17,1 \%$ \\
Moderado & $(18) 38,3 \%$ \\
Muito & $(12) 25,5 \%$ \\
Dispositivo de auxílio à marcha & \\
Nenhum & $(18) 38,3 \%$ \\
Andador & $(20) 42,5 \%$ \\
Utilizavam cadeira de rodas & $(6) 12,8 \%$ \\
\hline Bengala & $(3) 6,4 \%$ \\
\hline
\end{tabular}

$\mathrm{n}=$ número de idosos; $\%=$ percentagem de idosos 
Apenas 12,8\% $(n=6)$ dos idosos relataram ter sofrido quedas nos últimos seis meses e $80,9 \%(\mathrm{n}=38)$ tinham medo de cair. Dezoito $(38,3 \%)$ não utilizavam nenhum dispositivo de auxílio à marcha (tabela 2).

O desempenho no MEEM foi baixo, sendo que $93,3 \%(n=44)$ apresentaram valores abaixo da nota de corte para sua escolaridade. $\mathrm{O}$ escore médio do teste foi 14,6 \pm 6,3 (mediana 14,0). $\mathrm{Na}$ GDS, 59,6\%(n=28) obtiveram valores que sugerem possível quadro depressivo, em média os idosos apresentaram 6,26 $\pm 2,9$ pontos (tabela 3).

O tempo para realizar o teste de mobilidade funcional, o TUG, variou de 0,10 a 1,58 minutos (mediana 0,22), segundo a tabela 3.

Tabela 3 - Descrição das características da triagem cognitiva e de depressão e teste de mobilidade funcional. Belo Horizonte-MG, 2009.

\begin{tabular}{lcccc}
\hline \multicolumn{1}{c}{ Variável } & (n) $\%$ & Média \pm DP & Mediana & Min-máx \\
\hline MEEM & & & & \\
$\quad$ Sugestivo de alteração cognitiva & $(44)$ ) $93,3 \%$ & $13,79 \pm 5,7$ & 13,0 & $0-24$ \\
Não sugestivo de alteração cognitiva & $(3) 6,7 \%$ & $26,0 \pm 4,0$ & 26,0 & $22-30$ \\
GDS & & & & \\
Sugestivo de depressão & $(28) 59,6 \%$ & $8,18 \pm 1,7$ & 8,0 & $6-13$ \\
Sem sugestão de depressão & $(19) 40,4 \%$ & $5,23 \pm 3,0$ & 5,0 & $0-5$ \\
TUG (min.) & - & $-*$ & 0,22 & $0,1-1,58$ \\
\hline
\end{tabular}

$\mathrm{n}=$ número de idosos; \%= percentagem de idosos; $\mathrm{DP}=$ desvio padrão; Min-Máx: valor mínimo e valor máximo; MEEM: Mini exame do estado mental; GDS:escala de depressão geriátrica; TUG: Timed Up and Go; min.: minutos

* Não foram reportados os dados da média, pois a distribuição dos dados não foi normal.

A maioria dos idosos relatou ser independente para realizar as cinco atividades do Katz (banharse: $80,9 \%$; vestir-se: $83,0 \%$; uso do banheiro:
80,9\%; transferir-se: 87,2\%; micção: 59,6\%; evacuação: $61,7 \%$; alimentar-se: $93,6 \%$ ) e nenhum relatou a dependência completa (tabela 4). 
Tabela 4 - Descrição da realização das atividades de vida diária (Índice de Katz). Belo Horizonte-MG, 2009.

\begin{tabular}{|c|c|}
\hline Atividade & (n) $\%$ \\
\hline \multicolumn{2}{|l|}{ Banhar-se } \\
\hline Independente & (38) $80,9 \%$ \\
\hline Necessita de ajuda não humana & (8) $17,0 \%$ \\
\hline Necessita de ajuda humana & (1) $2,1 \%$ \\
\hline Dependência completa & (0) $0 \%$ \\
\hline \multicolumn{2}{|l|}{ Vestir-se } \\
\hline Independente & (39) $83,0 \%$ \\
\hline Necessita de ajuda não humana & (8) $17 \%$ \\
\hline Necessita de ajuda humana & (0) $0 \%$ \\
\hline Dependência completa & (0) $0 \%$ \\
\hline \multicolumn{2}{|l|}{ Uso do banheiro } \\
\hline Independente & (38) $80,9 \%$ \\
\hline Necessita de ajuda não humana & (7) $14,9 \%$ \\
\hline Necessita de ajuda humana & (2) $4,4 \%$ \\
\hline Dependência completa & (0) $0 \%$ \\
\hline \multicolumn{2}{|l|}{ Transferir-se } \\
\hline Independente & (41) $87,2 \%$ \\
\hline Necessita de ajuda não humana & (6) $12,8 \%$ \\
\hline Necessita de ajuda humana & (0) $0 \%$ \\
\hline Dependência completa & (0) $0 \%$ \\
\hline \multicolumn{2}{|l|}{ Micção } \\
\hline Independente & (28) $59,6 \%$ \\
\hline Necessita de ajuda não humana & (16) $34,0 \%$ \\
\hline Necessita de ajuda humana & (3) $6,4 \%$ \\
\hline Dependência completa & (0) $0 \%$ \\
\hline \multicolumn{2}{|l|}{ Evacuação } \\
\hline Independente & (29) $61,7 \%$ \\
\hline Necessita de ajuda não humana & (15) $31,9 \%$ \\
\hline Necessita de ajuda humana & (3) $6,4 \%$ \\
\hline Dependência completa & (0) $0 \%$ \\
\hline \multicolumn{2}{|l|}{ Alimentar-se } \\
\hline Independente & (44) $93,6 \%$ \\
\hline Necessita de ajuda não humana & (3) $6,4 \%$ \\
\hline Necessita de ajuda humana & (0) $0 \%$ \\
\hline Dependência completa & (0) $0 \%$ \\
\hline
\end{tabular}

$\mathrm{n}=$ número de idosos; $\%=$ percentagem de idosos 


\section{DISCUSSÃO}

No estudo verificou-se que, nos 47 idosos avaliados, a distribuição em relação ao sexo foi bastante semelhante, sendo encontrado pequeno predomínio do sexo feminino $(51,1 \%)$. Entretanto, este achado não é o frequentemente descrito na literatura, pois na maioria dos estudos revisados, o percentual da população feminina nas ILPI é muito superior ao número de homens. ${ }^{17-19}$ Pavan et al., ${ }^{18} \mathrm{em}$ estudo realizado em uma ILPI do Rio Grande do Sul, verificaram que dos 110 idosos residentes na instituição, $80 \%$ eram do sexo feminino. Este predomínio feminino é em geral explicado pelo fato de as mulheres viverem mais que os homens, logo têm maior possibilidade de vivenciar doenças e incapacidades; e ainda por serem em maior número. ${ }^{18,20}$ Além disso, as mulheres idosas experimentam uma probabilidade maior de ficarem viúvas e em situações socioeconômicas desvantajosas. $^{18}$

Entretanto, também foram encontrados dois estudos, um realizado em uma ILPI no Distrito Federal ${ }^{21}$ e o outro em Fortaleza, ${ }^{22}$ que verificaram predomínio do sexo masculino nos idosos residentes na ILPI, respectivamente, de 58,8\% e 50,6\%. Estes diferentes achados, descritos na literatura, podem ser reflexo da mudança da dinâmica familiar e da sociedade nos últimos $\operatorname{anos}^{5,6} \mathrm{e} / \mathrm{ou}$ pode estar nos mostrando uma diferença no perfil dos institucionalizados nas várias regiões do país.

O estudo mostra que a maioria dos idosos era solteira $(46,8 \%)$, não tinha filhos $(51,1 \%)$ e analfabeta $(25,5 \%)$ ou com baixa escolaridade $(59,6 \%)$. Os dados corroboram os outros estudos, que verificaram que a maioria dos idosos era solteira $\left(38,8 \%{ }^{22}\right.$ e $\left.59,6 \%\right),{ }^{21}$ analfabeta $(52,1 \%)$ ou de baixa escolaridade $(27,7 \%) .{ }^{21}$ Estas características muitas vezes são descritas como fatores de risco para institucionalização. A baixa escolaridade também é um achado muito frequente, ao se avaliar instituições filantrópicas, como foi o caso da presente pesquisa. ${ }^{23}$

O principal motivo de institucionalização declarado pelos idosos foi a busca de tratamento
$(29,8 \%)$, seguida de doença $(14,9 \%)$, fatores que de certa forma estão ligados. Este fato pode mostrar uma realidade que ainda enfrentamos no Brasil, que é a falta de infraestrutura para responder às demandas desse grupo etário, quanto a serviços domiciliares e ambulatoriais, programas específicos e recursos humanos adequados. ${ }^{24,25}$ Esse fato muitas vezes pode estar associado à dificuldade financeira de manter um doente em casa, pelos altos custos gerados por ele, e às demanda de um cuidador. ${ }^{24,25}$ Logo, muitas vezes a alternativa é a institucionalização do idoso.

A maioria dos idosos pesquisados referiu ter pelo menos uma doença crônica $(57,4 \%)$ e fazer uso de medicamentos (91,5\%). Outros estudos também mostram grande frequência de doenças crônicas e de utilização de pelo menos uma medicação nos idosos institucionalizados. Gonçalves et al. ${ }^{19}$ encontraram que $82,2 \%$ dos idosos tinham doenças crônicas e $83,3 \%$ utilizavam pelo menos uma medicação. No estudo feito por Menezes et al., ${ }^{26}$ verificou-se que 91,6\% dos idosos institucionalizados utilizavam pelo menos uma medicação. Esses achados podem ser explicados pela condição de saúde do idoso institucionalizado, que em geral é mais debilitado que idosos da comunidade.

Com relação à autopercepção de saúde, neste estudo $61,7 \%$ dos idosos institucionalizados consideraram sua saúde como boa. Este dado está de acordo com os resultados encontrados por Gonçalves et al., ${ }^{19}$ que também verificaram que a maioria dos idosos referiu ter boa saúde. Atualmente, a percepção de saúde, ou a autoavaliação da saúde, é um dos indicadores mais utilizados em pesquisas gerontológicas. Seu uso é justificado porque a pior percepção de saúde é um preditor robusto e consistente de mortalidade. ${ }^{27}$

Pouco mais da metade dos idosos $(51,1 \%)$ referiu sentir dores crônicas. Em outro estudo, também realizado com idosos institucionalizados, foi verificada elevada prevalência $(73,3 \%)$ de dor crônica entre os idosos e, ainda, foi verificado que essa dor interferia de maneira negativa na capacidade funcional dos idosos. ${ }^{28}$ Entretanto, há muitas evidências de que, apesar 
de a dor gerar consequências negativas, como comprometimento da deambulação, redução da socialização, diminuição da independência nas AVDs e redução global da qualidade de vida, a dor em idosos continua a ser subavaliada e subtratada. ${ }^{28,29}$

Apenas 12,8\% dos idosos relataram ter sofrido quedas. Este foi um achado inesperado, uma vez que a prevalência de quedas em idosos asilados é considerada alta, em torno de $37,2 \%{ }^{30}$ e $38,3 \% .{ }^{19}$ Os fatores que podem ter influenciado este resultado são o fato de a instituição ser toda adaptada para evitar a ocorrência de quedas e a média de idade dos idosos avaliados ser inferior aos demais estudos. ${ }^{19,30}$ A literatura tem demonstrado que a incidência dos eventos aumenta com o avanço da idade. ${ }^{30,31}$

Do total de idosos avaliados, $80,9 \%$ tinham medo de cair. $\mathrm{O}$ medo de queda também é um achado frequente entre os idosos institucionalizados, independentemente de terem ou não caído. Teixeira et al. ${ }^{32}$ verificaram, em seu estudo, que o medo de cair foi relatado por $82,35 \%$ dos idosos, sendo que no grupo que já sofreu quedas este percentual foi de 87,5\%, e dentre os idosos que não caíram, 77,8\%. O grande problema do medo de cair é que ele normalmente leva o idoso a restringir suas atividades, o que pode reduzir a função muscular, culminando em declínio funcional e risco aumentado de quedas. ${ }^{32}$

Dos idosos avaliados, $61,7 \%$ utilizavam dispositivo de auxílio à marcha, sendo que $42,5 \%$ usavam o andador, $12,8 \%$, a cadeira de rodas, e $6,4 \%$, a bengala. Dois estudos avaliaram a utilização do dispositivo de auxílio à marcha em idosos institucionalizados. A frequência de utilização do recurso variou muito entre os estudos, uma vez que um deles verificou que pequena porcentagem dos idosos utilizava os dispositivos $(14,8 \%)^{26}$ e o outro, 35,6\% dos idosos. ${ }^{19}$ Uma possível explicação para o baixo número de idosos utilizando o dispositivo, no estudo de Menezes et al., ${ }^{26}$ pode estar ligada ao fato de que a pesquisa excluiu os cadeirantes da amostra. As diferentes frequências de uso também podem estar refletindo as características físico-funcionais dos idosos residentes nas diferentes instituições, dado que a utilização do dispositivo tem a finalidade de promover a independência funcional dos idosos que outrora eram incapazes de realizar. ${ }^{33}$

A frequência da órtese utilizada também foi consideravelmente discrepante entre os estudos. Menezes et al. ${ }^{26}$ encontraram, em seu estudo, que do total de 95 idosos avaliados, $1,05 \%$ utilizava o andador e 13,7\%, a bengala. Já Gonçalves et al. ${ }^{19}$ encontraram, em uma amostra de 180 idosos, que 8,8\% usavam a cadeira de rodas para a locomoção, 7,2\%, o andador, $13,5 \%$, a bengala e 6,1\%, muletas. Estes diferentes achados podem estar relacionados à prescrição da órtese pelo profissional da ILPI ou, novamente, pela necessidade funcional do idoso, já que dependendo das características e condições dos idosos, indica-se determinado dispositivo de auxílio. ${ }^{33}$

A avaliação utilizando o MEEM mostrou que 93,3\% dos idosos apresentaram triagem positiva para alterações cognitivas. Estes dados se assemelham ao estudo realizado por Converso et al., ${ }^{23}$ no qual aproximadamente $80 \%$ dos idosos institucionalizados apresentaram déficits cognitivos. A média do escore do MEEM descrita no mesmo estudo foi de 13 pontos, aproximando-se da média encontrada nesta pesquisa, que foi 13,8 pontos.

A alteração cognitiva é um achado muito frequente nas ILPIs e é considerado um dos motivos de institucionalização do idoso, ${ }^{10,23,34}$ uma vez que seu comprometimento pode causar déficits de raciocínio, memória, comunicação, praxia, orientação espacial e personalidade, o que afeta diretamente seu convívio social. Essas alterações aumentam o risco idoso de perder sua independência, necessitando assim da ajuda permanente de terceiros, devido ao declínio físico e à dificuldade de interagir com outras pessoas. ${ }^{10,23,35}$

Através da GDS, verificou-se que 59,6\% dos idosos apresentam sintomas depressivos. Os valores encontrados no estudo foram relativamente superiores aos dados encontrados na literatura que utilizou o mesmo instrumento 
de rastreio GDS-15. ${ }^{26,36}$ Santana et al. ${ }^{36}$ e Menezes et al., ${ }^{26}$ encontraram, respectivamente, $21,1 \%$ e $37,9 \%$ de idosos com suspeita de depressão. Entretanto, o escore médio encontrado no presente estudo ( 8,1 pontos) foi inferior à média encontrada para mulheres (10,6 pontos) e homens (12,7 pontos) no estudo de Quadros Junior et al. ${ }^{34}$

O transtorno depressivo é causa importante de morbidade, sofrimento e incapacidade, e afeta sensivelmente a qualidade de vida do idoso. Os sintomas depressivos geralmente levam a comprometimentos funcionais, deixando o idoso sem autonomia e mais dependente na realização das atividades cotidianas, estando também relacionadas ao aumento do número de idosos acamados em ILPIs. ${ }^{31,34,36}$ Deve-se ressaltar que tais sintomas podem influenciar negativamente o nível de atividade física, assim como o desempenho em testes cognitivos. ${ }^{34}$ Alguns pesquisadores sugerem que a institucionalização é uma situação estressante e desencadeadora de depressão. ${ }^{36}$

A média do tempo gasto no teste de mobilidade funcional pelo desempenho, TUG, foi de 22 segundos, sendo que os resultados variaram de 0,1 a 1,58 minutos. Este resultado mostra que em média os idosos apresentam limitação da mobilidade, uma vez que escores acima de 20 segundos estão relacionados a idosos que podem estar apresentando alterações de equilíbrio, marcha e capacidade funcional. ${ }^{13}$ De acordo com o estudo de Shumway-Cook et al., ${ }^{37}$ um tempo maior que 14 segundos para realizar o teste TUG está relacionado a um alto risco de quedas. Estes dados mostram que, apesar dos baixos índices de quedas encontrados, em média os idosos avaliados se encontram em risco de cair e de terem alterações da capacidade funcional, o que reflete a necessidade de um trabalho preventivo para garantir a mobilidade.

Neste estudo também se verificou um amplo espectro de mobilidade funcional entre os idosos residentes na ILPI, uma vez que os resultados do TUG variaram de 10 segundos a 1, 58 minutos.
$\mathrm{Na}$ avaliação do desempenho das atividades básicas de vida diária pelo relato, utilizando o Índice de Katz, constatou-se que a maioria dos idosos era independente para realizar as sete tarefas. A capacidade de se alimentar sozinho foi a atividade com maior porcentagem de independência $(93,6 \%)$ e a micção, a atividade de menor frequência de independência (59,6\%). Nenhum idoso avaliado relatou ter dependência completa para realizar as atividades avaliadas. Guedes et al., ${ }^{38}$ utilizando o Índice de Barthel, também verificaram maior frequência de independência na alimentação $(62,38 \%)$ e menor porcentagem de independência na atividade de micção $(34,86 \%)$.

Em geral se evidencia uma hierarquia de perda da capacidade funcional nos idosos, mesmo em relação às atividades básicas. ${ }^{39}$ Como a alimentação é uma tarefa de cunho de subsistência realizada quase automaticamente pelos idosos, ela é preservada até uma fase mais final. ${ }^{10}$ Já a continência, em qualquer idade, depende não só daintegridade anatômica do trato urinário inferior e dos mecanismos fisiológicos envolvidos na estocagem e eliminação da urina, como também da capacidade cognitiva, mobilidade, destreza manual e motivação para ir ao toalete, ${ }^{10}$ por isso pode ser mais frequentemente acometida.

A alta independência dos idosos institucionalizados constatada no presente estudo pode evidenciar uma amostra com perfil diferenciado, ou seja, foram excluídos os idosos que apresentavam demência grave, limitações perceptivo-sensoriais graves (visão e audição) e os acamados - portanto, idosos com maior probabilidade de serem dependentes nas ABVDs. ${ }^{39}$

Mesmo nos resultados indicando ausência de dependência total dos idosos na instituição avaliada, vale ressaltar a importância e a necessidade de se encorajar os idosos no ambiente institucional, lançando mão de planos individualizados para estimular seu potencial de autocuidado, a fim de que permaneçam independentes o máximo de tempo possível, 
uma vez que as instituições são consideradas fatores que limitam sua independência. ${ }^{8}$

Uma possível limitação deste estudo estaria relacionada ao fato de a amostra ter sido de conveniência e de uma única ILPI, o que poderia limitar a generalização dos dados a indivíduos de outras ILPIs e regiões. Entretanto, apesar das limitações, o presente estudo mostra a necessidade de maior atenção aos idosos institucionalizados, tanto por parte de ações governamentais quanto privadas, a fim de que os idosos tenham melhor manutenção de sua capacidade funcional.

\section{REFERÊNCIAS}

1. Instituto Brasileiro de Geografia Estatística. (IBGE). Tábua completa de mortalidade - 2010. [Acesso em: 21 jan. 2012]; Disponível em: http://www.ibge.gov. br/home/estatistica/população/tabuadevida/2010/ notastecnicas.pdf.

2. Lima-costa MF, Loyola Filho A I, Matos DL. Tendências nas Condições de Saúde e Uso de Serviços de Saúde entre Idosos Brasileiros: um estudo baseado na Pesquisa Nacional por Amostra de Domicílios (1998, 2003). Cad. Saúde Pública. 2007; 23 (10): 2467-78.

3. Rosa TEC, Benício MHD'A, Latorre MRDO, Ramos LR. Fatores Determinantes na Capacidade Funcional dos Idosos. Rev. Saúde Pública. 2003; 37 (1): 40-8.

4. Fried LP, Ferrucci L, Darer J, Williamson JD, Anderson G. Untangling the Concepts of Disability, Frailty, and Comorbidity: implications for improved targeting and care. Journal of Gerontology: Medical Sciences. 2004; 59(3): 255-63.

5. Gaugler JE, Duval S, Anderson KA, Kane RL. Predicting Nursing Home Admission in the U.S: a meta-analysis. BMC Geriatrics. 2007; 7:13.

6. Perlini NMOG, Leite MT, Furini AC. Em Busca de Uma Instituição Para a Pessoa Idosa Morar: Motivos Apontados por Familiares. Rev. Esc. Enferm USP. 2007; 41(2): 229-236.

7. Aires M, Paz AA, Perosa CT. O Grau de Dependência e Características de Pessoas Idosas Institucionalizadas. RBCEH. 2006; 79-91.

8. Davim RMB, Torres GV, Dantas SMM, Lima VM. Estudo com Idosos de Instituições Asilares no Município de Natal/RN: características socioeconômicas de saúde. Rev. Latino-Am. Enfermagem. 2004;12(3): 518-524.

\section{CONCLUSÕES}

O perfil da população de idosos institucionalizados está de acordo com a literatura no que se refere ao sexo, estado civil, número de filhos e nível educacional. A maioria dos idosos apresentou alterações cognitivas e sintomas depressivos; entretanto, relataram ser independentes para as atividades básicas de vida diária, o que representa boa oportunidade para intervenções que visem prevenir ou minimizar possíveis perdas funcionais.

9. Montaño MBM, Ramos LR. Validity of The Portuguese Version of Clinical Dementia Rating, Rev. Saúde Pública, 2005;39(6):912-917.

10. Oliveira DLC, Goretti, LC, Pereira LSM. Performance in Daily Living Activities and Mobility Among Institutionalized Elderly People With Cognitive Impairments: pilot study. São Carlos, Rev. Bras. Fisioter. 2006;10(1):91-96.

11. Lino VTS, Pereira SRM, Camacho LAB, Ribeiro Filho, ST, Buksman S. Adaptação Transcultural da Escala de Independência em Atividades de Vida Diária (Escal de Katz). Cad. Saúde Pública. 2008; 24(1):103-112.

12. Duarte YAO, Andrade CL, Lebrão MLO. Index de Katz na Avaliação da Funcionalidade dos Idosos. Rev. Esc. Enferm. USP. 2007; 41(2): 317-25.

13. Podsiadlo D, Richardson S. The Timed "Up \& Go": a test of Basic Functional Mobility for Frail Elderly Persons. J Am Geriatr Soc. 1991;39:142-148.

14. Yesavage JA, et al. Development and validation of a geriatric screening scale. J Psychiatry Res. 1983; 17(1): 37-49.

15. Souza RL, Medeiros JGM, Moura ACL, Souza CLEM, Moreira IF. Validade e fidedignidade da Escala de Depressão Geriátrica na identificação de idosos deprimidos em um hospital geral. J. Bras. Psiquiatr. 2007; 56(2): 102-107.

16. Lourenço RA, Veras RP. Mini-exame do estado mental: características psicométricas em idosos ambulatoriais. Rev. Saúde Pública. 2006; 40(4): 712-719.

17. Carvalho MP, Luckaw ELT, Siqueira FV. Quedas e Fatores Associados em Idosos Institucionalizados no Município de Pelotas (RS, Brasil). Ciênc. Saúde Coletiva. 2011; 16 (6): 2945-2952. 
18. Pavan FJ, Meneghel SN, Junges JR. Mulheres Idosas Enfrentando a Institucionalização. Cad. Saúde Pública. 2008; 24 (9): 2187-2190.

19. Gonçalves LG, Vieira ST, Siqueira FV, Hallall PC. Prevalência de Quedas em Idosos Asilados do Município de Rio Grande, RS. Rev.Saúde Pública. 2008; 42 (5): 938-945.

20. Parahyba MI, Veras R, Melzer D. Incapacidade Funcional Entre as Mulheres Idosas no Brasil. Rev. Saúde Pública. 2005; 39 (3): 383-391.

21. Castellar JI, Karnikowski MGO, Viana LG, Nóbrega OT. Estudo da Farmacoterapia Prescrita a Idosos em Instituição de Longa Permanência. Acta Med Port. 2007; 20: 97-105.

22. Gaião LR, Almeida MEL, Heukelbach J. Perfil Epidemiológico da Cárie Dentária, Doença Periodontal, Uso e Necessidade de Prótese em Idosos Residentes em Uma Instituição na Cidade de Fortaleza, Ceará. Rev. Bras. Epidemiol. 2005; 8 (3): 316-323.

23. Converso MER, Iartelli I. Caracterização e Análise do Estado Mental e Funcional de Idosos Institucionalizados em Instituições Públicas de Longa Permanência. J. Bras. Psiquiatr. 2007; 56 (4): 267-272.

24. Lourenço RA, Martins CS, Sanchez MAS, Veras RP. Assistência Ambulatorial Geriátrica: Hierarquização da Demanda. Rev. Saúde Pública. 2005; 39 (2): 311-318.

25. Bessa MEP, Silva MJ. Motivações Para o Ingresso dos Idosos em Instituições de Longa Permanência e Processos Adaptativos: Um Estudo de Caso. Texto contexto - Enfermagem. 2008; 17 (2): 258-265.

26. Menezes RL, Bachion MM. Estudo da Presença de Fatores de Riscos Intrínsecos para Quedas em Idosos Institucionalizados. Ciênc. Saúde Coletiva. 2008; 13 (4): 1209-1218.

27. Lima-Costa MF, Peixoto SV, Matos DL, Firmo JOA, Uchôa E. A influência de Respondente Substituto na Percepção da Saúde de Idosos: um estudo baseado na Pesquisa Nacional por Amostra de Domicílios (1998, 2003) e na Coorte de Bambuí, Minas Gerais, Brasil Cad. Saúde Pública. 2007; 23 (8): 1893-1902.

28. Reis LA, Torres GV. Influência da Dor Crônica na Capacidade Funcional de Idosos Institucionalizados. Rev. Bras. Enferm. 2011; 64(2): 274-280.
29. Dellaroza MSG, Pimenta CAM, Matsuo T. Prevalência e Caracterização da Dor Crônica em Idosos Não Institucionalizados. Cad. Saúde Pública. 2007; 23 (5): 1151-1160.

30. Ferreira, DCO, Yoshitome AY. Prevalência e Características das Quedas de Idosos Institucionalizados. Rev. Bras. Enferm.2010; 63 (6): 991-997.

31. Valcarenghi RV, Santos SSC, Berlem ELD, Pelzer MT, Gomes GC, Lange C. Alterações na Funcionalidade/cognição e Depressão em Idosos Institucionalizados que Sofreram Quedas. Acta Paul. Enferm. 211; 24(6): 828-833.

32. Teixeira DC, Oliveira IL, Dias RC. Perfil Demográfico, Clínico e Funcional de Idosos Institucionalizados com História de Quedas. Revista Fisioterapia em Movimento. 2006; 19 (2): 101-108.

33. Hook FWVD, Weiss BD. Ambulatory Devices for Chronic Gait Disorders in the Elderly. American Family Physician. 2006; 67 (8): 1717-24.

34. Quadros Junior AC, Santos RF, Lamonato ACC, Toledo NAS, Coelho FGM, Gobbi S. Estudo do Nível de Atividade Física, Independência Funcional e Estado Cognitivo de Idosos Institucionalizados: Análise por Gênero. Brazilian Journal of Biomotricity. 2008; 2 (1): 39-50.

35. Gorzoni ML, Pires SL. Aspectos Clínicos da Demência Senil em Instituições Asilares. Rev. Psiquiatr Clínica. 2006; 33 (1): 18-23.

36. Santana AJ, Barboza Filho JC. Prevalência de Sintomas Depressivos em Idosos Institucionalizados na Cidade de Salvador. Revista Baiana de Saúde Pública. 2007; 31 (1):134-146.

37. Shumway-Cook A, Brauer S, Woollacott M. Predicting the Probability for Falls in CommunityDwelling Older Adults Using the Timed Up \& Go Test. Phys Ther. 2000; 80 (9): 896-903.

38. Guedes JM, Silveira RC. Análise da Capacidade Funcional da População Geriátrica Institucionalizada na Cidade de Passo Fundo - RS. Revista Brasileira de Ciências do Envelhecimento Humano. 2004; 10-21.

39. Marra TA, Pereira LSM, Faria CDCM, Martins MAA, Tirado MGA. Evaluation of the activities of daily living of elderly people with different levels of dementia. Rev. Bras. Fisioterapia, 2007; 11 (4): 267-273. 